\title{
Post-Acute Care as a Key Component in a Healthcare System for Older Adults
}

\author{
Yu-Chun Wang ${ }^{1}$, Ming-Yueh Chou ${ }^{1,2,3}$, Chih-Kuang Liang ${ }^{1,2,3}$, Li-Ning Peng ${ }^{2,3,4}$, Liang-Kung Chen ${ }^{2,3,4}$, Ching-Hui Loh ${ }^{3,5,6}$ \\ ${ }^{1}$ Center for Geriatrics and Gerontology, Kaohsiung Veterans General Hospital, Kaohsiung, Taiwan \\ ${ }^{2}$ Aging and Health Research Center, National Yang Ming University, Taipei, Taiwan \\ ${ }^{3}$ Department of Geriatric Medicine, National Yang Ming University, Taipei, Taiwan \\ ${ }^{4}$ Center for Geriatrics and Gerontology, Taipei Veterans General Hospital, Taipei, Taiwan \\ ${ }^{5}$ Center for Aging and Community Health, Hualien Tzu Chi Hospital, Hualien, Taiwan \\ ${ }^{6}$ School of Medicine, Tzu Chi University, Hualien, Taiwan
}

Corresponding Author:

Ching-Hui Loh, MD, PhD

https://orcid.org/0000-0002-3324-0962

Center for Aging and Community

Health, Hualien Tzu Chi Hospital, 707,

Sec. 3, Chung-Yang Rd., Hualien 970,

Taiwan

E-mail: twdoc1960@gmail.com

Received: February 19, 2019

Revised: March 23, 2019

Accepted: March 26, 2019
Older adults often experience functional decline following acute medical care. This functional decline may lead to permanent disability, which will increase the burden on the medical and long-term care systems, families, and society as a whole. Post-acute care aims to promote the functional recovery of older adults, prevent unnecessary hospital readmission, and avoid premature admission to a long-term care facility. Research has shown that post-acute care is a cost-effective service model, with both the hospital-athome and community hospital post-acute care models being highly effective. This paper describes the post-acute care models of the United States and the United Kingdom and uses the example of Taiwan's highly effective post-acute care system to explain the benefits and importance of post-acute care. In the face of rapid demographic aging and smaller household size, a post-acute care system can lower medical costs and improve the health of older adults after hospitalization. (Ann Geriatr Med Res 2019;23:54-62)

Key Words: Post-acute care, Elderly, Referrals, Intermediate care facilities, Interdisciplinary health team

\section{INTRODUCTION}

Post-acute care is a key element of the healthcare system for older adults and an important strategy for maintaining their health. Compared with younger adults, older adults are more likely to have various comorbidities and disabilities and typically require a longer time to recover from acute illness. Cost considerations strictly limit the number of days of hospitalization, which limits the opportunities for older adults to regain their health following illness. Post-acute care models were therefore developed to meet the need of older adults for post-illness recovery. An important aspect of post-acute care is treatment outside a medical center, which enables patients to continue to receive appropriate care after completing treatment for an acute illness and to help them regain the autonomy they enjoyed before hospitalization.

Compared with the general adult population, older adults have a decreased physiological reserve and take longer to recover from illness. Apart from requiring more time for recovery, older adults need to return to a state of functional autonomy to reduce the incidence of subsequent disability, thus improving their quality of life and reducing the long-term burden on the care system. Previous research found that as many as 25\%-35\% of older adults lose at least one activity of daily living (ADL) following 10-14 days of hospitalization for acute illness, ${ }^{1,2)}$ 25\% suffer cognitive functional decline after 10-14 days of hospitalization, ${ }^{3,4)}$ and 20\%-25\% experience emotional disturbances after discharge (chiefly depression and anxiety). ${ }^{5,6)}$ In addition, in $20 \%-40 \%$ of such patients, their nutritional status is worse than it was before hospitalization, ${ }^{7-9)}$ and all were at risk for falls after discharge. ${ }^{10)}$ However, the vast majority of patients discharged after acute care return home with no further care. A study showed that older adults who cannot recover their cognitive and physical function after hospitalization for an acute illness are at significantly greater risk of rehospitalization, disability, mortality, and placement in a long-term care facility; in addition, this places an increased care burden on family members. ${ }^{10)}$ The World Health Organization 2015 World Report on Aging and Health states that different medical systems will face different challenges as demographic aging increases the need for care. Despite the fact that developed countries have well-established medical systems, a fragmented care system has become a difficult- 
to-avoid phenomenon as a country's population ages. The insufficient medical systems of developing countries are also struggling to respond to aging. ${ }^{11)}$

This report reveals that the healthcare system as a whole still focuses on specialized care rather than a holistic return to health, provides chiefly fragmentary care, and commonly neglects providing care plans that enable functional recovery in older adults following treatment for acute illness. Consequently, in order to broadly enhance the quality of care for older adults and avoid a large increase in the disabled population due to aging, it is essential to construct a post-acute care service model for older people after hospitalization. The post-acute care model has been implemented in different countries for decades, but thus far, there is no consensus on an international definition. Several related terms need to be clearly distinguished, including transitional care, sub-acute care, post-acute care, and intermediate care. Transitional care broadly refers to the coordination and continuity of healthcare as patients transfer between different levels of care within the same location (such as acute illness to chronic illness or deconditioning status to permanent dependent status) or between different locations, including transfer between different hospitals, the patient's home, primary and specialty care offices, long-term care facilities, sub-acute units, and post-acute units. The model of sub-acute care being conducted in the American healthcare system focuses on the extension of acute medical services beyond the period of acute instability by a non-acute care unit within or close to the hospital in order to shorten the length of acute inpatient care. Furthermore, the model of post-acute care in the American healthcare system is defined as providing integrated inpatient care directed at persons after their acute condition is stabilized by dealing with one or more specific, complex medical conditions in order to improve care quality and facilitate a return to the community. Meanwhile, the model of intermediate care in British healthcare systems is aimed at older people at home or in well-designed care facilities, in which medical and rehabilitation services are intended to promote autonomous living functions, reduce unnecessary hospitalization, ensure returning to home at an early date, and avoid unnecessary placement in a long-term care facility.

This paper therefore reviews the post-acute care development process, definitions, and care goals as well as the predominant post-acute care models in the United Kingdom (UK) and the United States (US). Lastly, this paper describes current post-acute care developments in Taiwan, assesses their effectiveness, proposes ideas for improving the post-acute care system in Taiwan, and provides some conclusions.

\section{DEVELOPMENT OF POST-ACUTE CARE}

The development of post-acute care in the medical systems of various countries was originally motivated largely by the desire to reduce the length of acute care hospitalization and thereby lessen overall medical expenditures. Many countries consequently developed post-acute care models based on this consideration. For instance, the US began providing Medicare and Medicaid in $1965,{ }^{12,13)}$ and the diagnosis-related group (DRG) concept was developed during the 1960s by Yale University scholars Fetter and Thompson ${ }^{14)}$ to promote disease management by classifying severity in diseases of the same type. DRG systems rely on the concept of "case grouping" to establish disease classification systems, and a DRG approach was adopted as a health insurance pricing standard when the US Congress passed the Medicare Prospective Payment System in 1983. However, because this approach allows uniform payments for different conditions, hospitals were encouraged to reduce acute medical expenditures and days of acute hospitalization and therefore had an incentive to discharge patients prematurely or refer them prematurely to other types of care. ${ }^{15,16)}$ As a result, the US began promoting sub-acute care during the 1980s. Sub-acute care, originally proposed to control the number of days of acute hospitalization, was also intended to meet patients' care needs after discharge and chiefly relied on medical units or skilled nursing facilities (SNFs) near hospitals to provide care. Nevertheless, although sub-acute care was actively promoted to reduce acute medical expenditures, it failed to improve the quality of care as a whole. To enable patients with stable conditions to obtain early discharge and receive active post-acute care via a comprehensive care approach, the US 1997 Balanced Budget Act expanded the post-acute care services covered by Medicare, leading to the establishment of four key types of post-acute care units, namely SNFs, home health agencies (HHAs), inpatient rehabilitation facilities (IRFs), and long-term care hospitals (LTCHs), which covered inpatient, institutional, residential, and active rehabilitation care models. The American post-acute care case acceptance standards based on the post-acute care DRG systems led to significantly different groups of patients receiving care than under the intermediate care system of the UK. ${ }^{17,18)}$

Although numerous studies have confirmed that aging will increase medical expenditures, ${ }^{19)}$ National Beds Inquiry report of the UK in 2000 found that, despite the continuous increase in the country's older population starting in the 1980s, the number of acute hospital beds and days of patient hospitalization has in fact fallen steadily. The strict control of the number of days of hospitalization led to a very high percentage of older adults whose cognitive and physical function had not appropriately recovered being transferred to long-term care facilities. Because this system both decreased the health of the older adults and also burdened the country's long-term care system, the UK was forced to establish a new integrated healthcare service model to provide care during the post-acute period to aid in the recovery of physical function. This model was termed the intermediate care system. The UK began devel- 
oping intermediate care in 2001; it is currently considered one of the main reform programs of the British National Health Service and serves as an important basis for healthcare in the Department of Health and Social Care's National Service Framework for Older People. Intermediate care has been the most important policy affecting care for older adults in the UK in recent years.

Intermediate care and post-acute care are both forms of transitional care for patients returning to the community after hospitalization for acute conditions, and both integrate community care resources to provide patients the needed healthcare services after they return home. Even though the American and British healthcare systems employ different assessment criteria and care service systems, intermediate care and post-acute care are based on the same concepts and, to avoid confusion, this paper will uniformly use post-acute care to refer to the care provided under both systems.

\section{POST-ACUTE CARE DEFINITIONS AND GOALS}

Despite the significant importance of post-acute care to older adults, there is no uniform international definition of post-acute care thus far, and this concept has been defined in different ways.

\section{American Definition}

The US Joint Commission on Accreditation of Healthcare Organizations and the American Healthcare Association have jointly defined sub-acute care as "integrated inpatient care directed at persons with all acute illnesses, accidental injuries, or serious diseases. It involves goal-directed treatment, does not depend on advanced technology, testing, or complex diagnostic procedures, and provides patients with alternatives to acute inpatient treatment after their acute conditions have stabilized by dealing with one or more specific, complex medical conditions or providing complex technical treatment."20) As a consequence, early sub-acute care was chiefly aimed at patients who had passed the period of acute instability but still required diagnostic or continuing therapeutic services. Here, post-acute care was defined as a series of services provided to patients whose conditions had stabilized following acute hospitalization in order to facilitate their return to the community; these services could include medical treatment, nursing care, rehabilitation, and residential care. Because the definitions and service models of sub-acute care and post-acute care overlap to some extent, after the expansion in coverage of post-acute care under Medicare following the passage of the Balanced Budget Act in 1997, the Medicare system has uniformly used the term post-acute care. The early development of sub-acute care in the US chiefly focused on extending acute medical services provided to patients by a non-acute care unit within or close to the hospital to which they had been admitted. Although the provision of such still-incomplete medical care services shortened the length of acute inpatient care, and thereby reduced medical expenditures, subsequent efforts to improve post-acute care were sought to improve the quality of care and facilitate return to the community.

\section{British Definition}

The development of post-acute care in the UK was based on national policy before evidence was available regarding its effectiveness. ${ }^{21)}$ National Service Framework for Older People is defined as "a new medical and rehabilitation service aimed at bridging the gap between hospital care and the community care system." Within this framework, post-acute care is further described as "a new care service provided to the older people at home or in well-designed care facilities, in which service from trained care teams promotes autonomous living functions, reduces unnecessary hospitalization, and ensures that older adults can be discharged and return home at an early date, and avoid unnecessary placement in a long-term care facility."22)

The British Geriatric Society defines post-acute care as follows. ${ }^{23)}$ (1) The post-acute care model is designed to assist patients to transition from illness to recovery while avoiding placement in a long-term care facility of patients who could receive care at home. (2) Post-acute care relies on a series of care services to help patients return home successfully after hospital discharge to aid the transition from recovery to functional autonomy. The chief goals of post-acute care extend beyond medical aspects to encompass the provision of appropriate transitional integrated care to patients with the potential for functional recovery, thereby facilitating their return to their place of residence. (3) The chief content of post-acute care services is aimed at patients with multiple care needs; although medical center resources do not provide such care, because services may exceed the scope of primary-level care, they must be provided via a team model.

\section{Goals of Post-Acute Care}

From a practical perspective, post-acute care must comply with the following five goals. (1) Care recipients must be patients not requiring long-term hospitalization or premature placement in a long-term care facility. (2) The services provided should be based on a comprehensive assessment of the older patient and individualized treatment plans drafted in accordance with the results of such assessments. (3) As much as possible, such service should seek to enhance patients' independent, autonomous living ability and have the ultimate goal of allowing patients to live independently at home. (4) Post-acute care services possess time restrictions, will not exceed 6 weeks as a rule, and will usually last no more than 2 weeks. (5) Although the content of services must encompass all treatment areas, there must be individual assessment mechanisms, individual case history records, and case history sharing procedures.

In summary, post-acute care must have clear goals 
(avoid unnecessary hospitalization and enhance independence and autonomy), integrated comprehensive assessments, clear cognitive and physical functional recovery therapeutic regimens, clear service time limits (to avoid confusion with long-term care), and joint implementation involving different units (hospitals, primary care units, and social welfare units). The goals of British post-acute care are not identical to those of sub-acute care in the US, as post-acute care constitutes a larger-scale, active service model integrating the resources of acute care hospitals, community hospitals, primary-level medical units, community public health units, and social welfare units. Postacute care is intended not only to reduce the use of acute hospital beds but also to allow frail older adults to age in place.

\section{POST-ACUTE CARE MODELS}

The optimal post-acute follow-up care setting for older adults depends on the individual patient's treatment goals, and a single care setting is not necessarily the best choice. Because the chief goal of post-acute care is to promote independent living and avoid unnecessary hospitalization, a series of comprehensive care services is employed to help patients return home in the best possible state of health. Post-acute care services should therefore take "care close to home" as a principle and extend certain medical and social welfare services to the communities in which patients and their families live. Because patients' needs span different organizations and services, multiple professional groups or organizations must take responsibility for postacute care. The post-acute care setting should emphasize patient-centered care, integrate various kinds of service resources, and thereby seek to promote the greatest possible degree of cognitive and physical functional recovery. An additional important goal of post-acute care is to ensure that patients are not unnecessarily placed in a longterm care facility after hospital discharge. To achieve this goal and ensure patients can return to independent living, a physical reablement program must be arranged for patients before their return home.

Since the development of post-acute care, various settings and models have been introduced to achieve its goals. ${ }^{24)}$ In the US, SNFs, HHAs, IRFs, and LTCHs have emerged to fill this need. ${ }^{20)}$ In British post-acute care model, community hospitals, hospitals-at-home, nurseled units, day hospitals, and social services all play roles. ${ }^{25)}$ Service team models include rapid response teams, hospital supported discharge teams, community assessment and rehabilitation schemes, home rehabilitation teams, and stroke rehabilitation outreach teams. ${ }^{25)}$

The following are some of the most commonly seen post-acute care service models in the UK and the US.

\section{British Post-Acute Care Service Models}

\section{Home-based post-acute care services}

Rapid response teams: Quick assessment and diagnosis of patients at home, rapid drafting of treatment programs for resident patients, and reliance on various kinds of hospital-at-home care and care services to reduce the risk of hospitalization.

Hospital supported discharge team: Reliance on shortterm hospital-at-home services or other life care services to enable patients to quickly return home from acute hospitals and ensure they continue to receive comprehensive post-discharge care until able to live independently.

Hospital-at-home: Hospitals-at-home provide intensive treatment services in a residential environment, including tests and treatments ordinarily provided only in hospitals or clinics, in order to reduce the need for hospitalization; they can also provide patients with seamless post-discharge treatment services. Hospitals-at-home can further provide postoperative patients with the same treatments patients receive in hospitals, including wound care, tube replacement, and residential rehabilitation, which enables patients to recover their health at home.

\section{Residential post-acute care services}

Residential rehabilitation: Patients receive rehabilitation while in short-term residence at a care organization, where they can improve their cognitive and physical functions to the point where they can lead independent lives. Care organizations may include community hospitals, rehabilitation centers, nursing homes, or long-term care facilities. Residential care patients must have a stable condition but require short-term intensive rehabilitation to recover their ADLs and must be considered potentially able to live at home. Treatment time ranges from 1 to 2 weeks for short stays (such as after pneumonia) to 4 to 6 weeks for longer stays (such as after major surgery), and stays may be appropriately extended when the situation warrants.

Day rehabilitation: Patients visit day hospitals or care centers to continue to receive daytime rehabilitation services.

Community hospitals: These differ from medical centers in that community hospitals accept patients whose condition has stabilized but continue to have multiple care needs. Community hospitals chiefly focus on physical reablement as well as providing other relevant medical and nursing treatments.

Although British care services encompass various models reflecting different areas and needs, in principle, patients are not accepted according to the diagnosis, but instead, according to their care needs and functional impairment due to acute issues. Although these care models can be classified as bed-based or home-based, their core service involves the provision of assistance from an interdisciplinary team after hospital discharge. In a 2009 summary analysis of the various models, Young ${ }^{25)}$ found 
that hospital-at-home had the greatest empirical evidence of benefit. Apart from reducing expenditures, hospitals-athome also reduced the number of days of hospitalization and the rehospitalization rate, enhanced overall functional ability, and allowed patients to avoid placement in a longterm care facility. Community hospitals were found to be as effective as large hospitals ${ }^{26}$ but had greater cost effectiveness. Although empirical evidence was insufficient to assess the other models, they appeared to have similar effectiveness, with the biggest difference being that nurseled units had a longer length of stay than other models. ${ }^{27}$ 2012 National Audit of Intermediate Care reviewed the literature and actual implementation data ${ }^{28)}$ and performed a comparative analysis of residential care and home-based care; although the acceptance criteria were similar, the medical issues of patients receiving residential care tended to be more complex than those of patients receiving home-based care, and the residential care system also had more interdisciplinary care personnel and more resources. In general, the overall cost of home-based care was approximately one-fourth that of residential care, but both types of care allowed over $60 \%$ of patients to continue living at home (66.5\% vs. $68.5 \%$, respectively), and both had similar mortality rates $(3.0 \%$ vs. $2.4 \%$, respectively). This audit report also recommended that, despite the individual focus of the different models, they did not need to exist independently, and their mutual integration and linkage should be considered. In addition to analysis of expenditures and consumption of medical resources, the audit noted a lack of appropriate prognosis assessment indicators.

\section{American Post-Acute Care Models}

\section{IRFs}

Rehabilitation facilities established within hospitals primarily to provide rehabilitation for 13 types of disease requiring follow-up rehabilitation, including stroke, spinal injury, traumatic brain injury, limb amputation, and hip fracture. The goal of these facilities is to provide rehabilitation treatment to patients requiring at least $3 \mathrm{~h}$ of rehabilitation each day because of disabilities and functional impairments resulting from acute illness; such patients have an average of 12 days of hospitalization.

\section{SNFS}

Established within nursing homes, SNFs are reimbursed by Medicare and provide integrated services to patients. Accepted cases must undergo assessment employing the US Resident Assessment Instrument and comply with the Resource Utilization Groups classification of the longterm care case mix index system. Services provided by SNFs include physician's rounds, rehabilitation, nutrition care, and skilled nursing measures such as drug treatment, wound treatment, and ventilator care. However, everyday caretaking services are not included within the scope of reimbursement at these facilities.

\section{HHAs}

Home-based care cases must undergo a physician's assessment and comply with the Home Health Resource Groups classification before acceptance. HHAs provide skilled nursing care, physical therapy, functional and language therapy, social work services, and other healthcare services to patients at home.

\section{LTCHS}

LTCHs established within hospitals or existing independently can meet the needs of complex patients who have left the acute instability stage but still have multiple comorbidities or multiple care needs and require hospitalization and assistance from a medical team. Typical situations include ventilator care or weaning, complex wound treatment, cardiopulmonary failure, or septicemia still requiring treatment. After an interdisciplinary team helps to stabilize the patient, different post-acute care units or long-term care services take over.

Post-acute care is reimbursed by Medicare in the US. In the early days, different care models were employed in cases with different diseases and different functional and care needs. However, because the assessment instruments and payment conditions differ according to the model used, procedures have become exceedingly complex, with differences between patients adding to the complexity. As a result, although post-acute care has eased the burden on acute care departments and reduced the number of days of hospitalization, it is difficult to perform any meaningful comparison of individual care models. As pointed out by the New England Journal of Medicine in 2014 and JAMA Internal Medicine in 2015, ${ }^{29,30)}$ although the establishment of post-acute care models has reduced the number of days of hospitalization in an acute ward, it has also led to a skyrocketing number of days of post-acute care and postacute care expenditures. In addition, because acute units and post-acute care models function independently, but allow mutual referral, and because their payment models also differ, it is difficult to assess overall care quality and effectiveness or to increase cooperation or quality of care system-wide.

\section{DEVELOPMENT OF POST-ACUTE CARE IN TAIWAN}

\section{The Post-Acute Care Development Process}

The average age of veterans in Taiwan is approximately 69 years, and 12\% of veterans are single. Veterans living in a veterans' home have an average age of 85 years, and most have complex medical needs, including comorbidities. $^{31)}$ Because of the needs of these older veterans, Taiwan has had to develop a superior care model providing integrated follow-up care in order to improve the quality of healthcare received by older veterans, help hospitals promptly and safely discharge them, reduce their shortterm rehospitalization rate, and reduce the need for early 
placement in a long-term care facility owing to disability.

Past medical systems, chiefly geared toward the treatment of disease, could not deal with every healthcare need of older adults, and such systems also lacked holistic care for the frail older patient. A British study found that community hospitals offer trustworthy post-acute care, and quality of life improves the most when patients receive care at nearby community hospitals. ${ }^{26)}$ As a consequence, post-acute care has been promoted largely based on the community hospital system. In Taiwan, the development of post-acute care has followed a dual strategy in which community medical teams have been enlisted to help community hospitals develop post-acute care, while also encouraging hospitals to promote a community home-based health model. The result has been equal attention paid to the development of both community hospital and homebased models.

Starting in June 2007, the veteran's medical system took the lead in establishing a post-acute care service network. Initially, Taipei Veterans General Hospital launched a trial program in community hospitals established by its Taoyuan and Yuanshan branch community hospitals. Under this program, older veterans were assessed by a geriatric medicine team when discharged from Taipei Veterans General Hospital and were transferred to one of two community hospitals for follow-up treatment if the assessment indicated a potential for recovery. The two branches also established interdisciplinary geriatric care teams, including attending physicians, nurses, case managers, social workers, rehabilitation specialists, physical therapists, occupational therapists, nutritionists, and pharmacists, who perform comprehensive assessments within $24 \mathrm{~h}$ of a patient's arrival. The teams also draft treatment plans based on the results of comprehensive assessment and regularly hold team conferences to discuss each patient's recovery progress and needed treatment. The teams actively implement 4-week functional recovery plans (to a maximum of 12 weeks); when patients regain their ability to live independently, arrangements are made for them to return home (Fig. 1). Because the trial program proved to be highly effective, post-acute care centers were established in central (chiefly by Taichung Veterans General Hospital) and southern Taiwan (chiefly by Kaohsiung Veterans General Hospital), creating a network of five community veteran's hospitals providing post-acute care.

\section{Assessment of Post-Acute Care Needs}

The provision of effective post-acute care services depends on the seamless linkage of different care units, and the most important step is the assessment of patient-centered, post-acute care needs during hospitalization. In the UK, assessment of most post-acute care needs is performed via judgment of individual patients by medical center care personnel, with the key aspects being whether patients

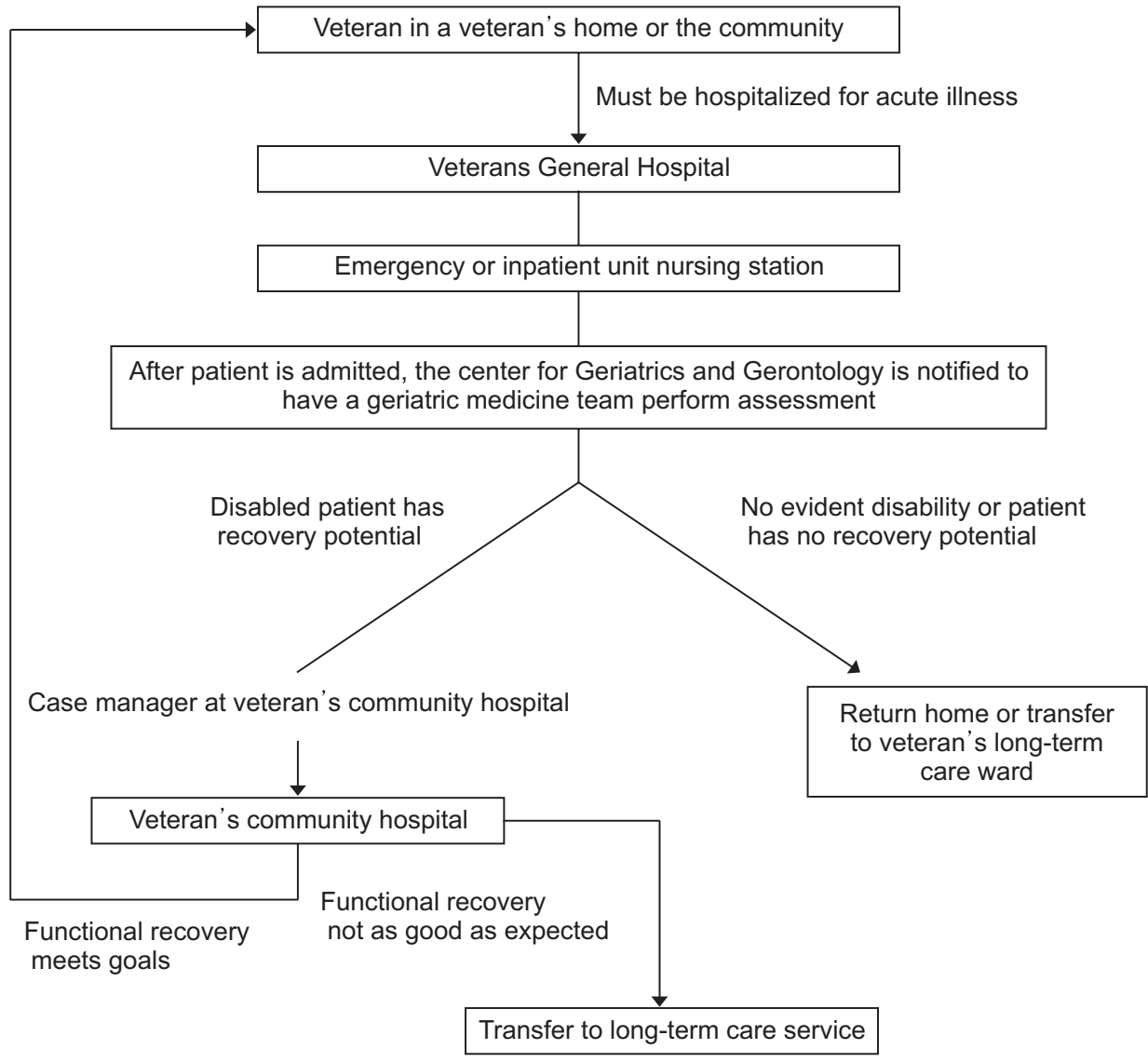

Fig. 1. Flowchart of post-acute care implementation procedures in the Veteran's Medical System in Taiwan. 
exhibited functional decline during hospitalization and whether they have the potential for functional recovery; disease diagnosis is not the sole assessment criterion. A study showed that approximately $25.8 \%$ of older adults need post-acute care after hospitalization, and $20.7 \%$ of this care is connected to rehabilitation. ${ }^{32)}$ Risk factors for functional decline during hospitalization include older age, low socioeconomic status, disability, poor cognitive function, delirium, multiple comorbidities, use of multiple drugs, history of falls, and geriatric syndrome. ${ }^{33-36)}$ A medical center in Taiwan found that 25.2\% of 409 older adults (average age: 80.4 years) who underwent post-acute care needs assessment had post-acute care needs, a rate similar to those found by British needs surveys. Among those referred to post-acute care, 97.7\% had motor impairments, 90.7\% were at risk for poor nutrition, $74.4 \%$ took multiple medications, 53.3\% had cognitive impairments, 41.9\% had pain issues, 39.5\% had a sleep disorder, 32.6\% had visual impairments, $14.0 \%$ had depressive symptoms, and $7.0 \%$ were at risk for pressure injuries. ${ }^{37)}$ Because of this wide range of issues, post-acute care needs cannot be assessed on the basis of disease diagnosis alone; the state of a patient's functioning must also be considered. Because patients entering post-acute care tend to have complex clinical needs, care plans must be based on the results of functional assessment, which will ensure that care plans address the needs of the individual patient and are revised based on changes in the patient's health status.

The timing of needs assessment is a key aspect of postacute care. Because post-acute care is usually connected with DRG payments in the US, assessment of needs and referral are relatively straightforward. Nevertheless, needs assessment must begin long before hospital discharge. The key to successful post-acute care is screening for the patient's functional recovery potential. In addition, for patients with multiple, complex care needs, selecting the optimal post-acute care remains a challenge.

\section{EFFECTIVENESS AND QUALITY OF POST-ACUTE CARE IN TAIWAN}

Although British studies have found that post-acute care can effectively reduce the rate of rehospitalization and placement in long-term care facilities, these studies made little mention of individual treatment results and the burden on caregivers. ${ }^{30,37-39)}$ Chen et al. $^{40)}$ found that postacute care can indeed be effective in assisting patients' functional recovery and can reduce 1-year mortality by as much as $62 \%$. Post-acute care can effectively boost patients' ADLs, instrumental ADLs, and cognition function, while reducing symptoms of pain and depression. Furthermore, Lee et al. ${ }^{41}$ discovered a significant correlation between physical therapy and functional recovery during the post-acute care period, such that the more physical therapy the patient received, the better the functional recovery achieved, along with easing of pain and improve- ment in depression symptoms. Although sex, home living arrangement, and basic physical functioning are correlated with functional recovery, the most important factor influencing the effectiveness of post-acute care is the patient's cognitive function. ${ }^{42)}$

A 4-year longitudinal study of patients with hip fractures by Peng et al. $^{43)}$ found that if patients received inhome or community hospital post-acute care following surgery, motor function could be recovered within a short time and long-term mortality would drop significantly. Liu et al. ${ }^{44)}$ found that, although 38\% and 43\% of patients in post-acute care units had symptoms of depression and anxiety, respectively, after integrated team treatment, symptoms of depression improved along with physical function, and the original symptoms of depression did not affect their prognosis.

When patients receive post-acute care services, unplanned rehospitalization occurs in $4 \%-35 \%$ of cases. ${ }^{45}$ ) Rehospitalization usually occurs in the first few days of post-acute care, and $89 \%$ of all cases are a result of infection, chronic obstructive pulmonary disease, acute heart attack, or heart failure. ${ }^{46)}$ We found that only approximately $12.9 \%$ of the patients are transferred to the acute ward, which is better than the figures for many European and American countries, and the reason for hospitalization is usually infectious disease. ${ }^{46)}$

Taiwan's National Health Insurance began implementing post-acute care services for stroke patients in 2014. In the community hospital post-acute care model for stroke patients, personnel provide high-intensity rehabilitation training based on the patient's Comprehensive Geriatric Assessment results, and patients are accepted based on the occurrence of stroke and a modified Rankin Scale Score of 2-4, indicating the patient's functional status as determined by the acute hospital diagnosis. Interdisciplinary team-based post-acute care, which lasts up to 12 weeks, significantly improves patients' physical functioning, cognitive function, mood, nutritional status, and neurological function, particularly balance. ${ }^{47)}$ A study tracking patients discharged from the hospital to post-acute care found that patients continue to improve following discharge home and have lower rates of post-discharge emergency care and risk of rehospitalization within 90 days compared with those who did not receive post-acute care. ${ }^{43)}$

\section{FUTURE OUTLOOK AND CONCLUSIONS}

Demographic aging has increased the number of older adults experiencing significant functional decline following acute medical care; if effective intervention for these short-term disabilities is not implemented, these patients are at greater risk of rehospitalization or placement in a long-term care facility, burdening the medical system, patients' families, and society as a whole. In the midst of rapid aging and a trend toward smaller families, the establishment of a post-acute care service network will not 
only reduce medical expenditures and the burden on the long-term care system but can also help older adults effectively maintain self-care and their quality of life. How to integrate existing resources effectively to optimize postacute care is consequently an important issue. Supported by the effectiveness of the stroke post-acute care provided since 2014, Taiwan's National Health Insurance began implementing a number of post-acute care service programs in 2017, including programs for frail seniors and patients with heart failure, traumatic brain injury, and fragility fractures. It has also encouraged the establishment of various post-acute integrated care models, including an inpatient model, day care model, and home-based model. These models will enable older adults to receive optimal functional recovery care and maintain the best possible quality of life. Apart from its proven economic effectiveness, the evidence-based community home-based postacute care models being implemented in Taiwan can also enable older individuals to receive rehabilitation at community health centers near their homes, or even at home. Because of this greater use of non-hospital resources, the implementation of the community hospital and homebased models of post-acute care will benefit all: the older people, their family members, the medical system, and the country as a whole.

\section{CONFLICTS OF INTEREST DISCLOSURES}

The researchers claim no conflicts of interest.

\section{REFERENCES}

1. Sager MA, Franke T, Inouye SK, Landefeld CS, Morgan TM, Rudberg MA, et al. Functional outcomes of acute medical illness and hospitalization in older persons. Arch Intern Med 1996;156:64552.

2. Inouye SK, Wagner DR, Acampora D, Horwitz RI, Cooney LM Jr, Hurst LD, et al. A predictive index for functional decline in hospitalized elderly medical patients. J Gen Intern Med 1993;8:645-52.

3. Murray AM, Levkoff SE, Wetle TT, Beckett L, Cleary PD, Schor JD, et al. Acute delirium and functional decline in the hospitalized elderly patient. J Gerontol 1993;48:M181-6.

4. Inouye SK, Viscoli CM, Horwitz RI, Hurst LD, Tinetti ME. A predictive model for delirium in hospitalized elderly medical patients based on admission characteristics. Ann Intern Med 1993;119:474-81.

5. Alamri SH, Bari AI, Ali AT. Depression and associated factors in hospitalized elderly: a cross-sectional study in a Saudi teaching hospital. Ann Saudi Med 2017;37:122-9.

6. Helvik AS, Engedal K, Krokstad S, Stordal E, Selbæk G. A comparison of depressive symptoms in elderly medical inpatients and the elderly in a population-based health study (the Nord-Trøndelag Health Study 3). Nord J Psychiatry 2012;66:189-97.

7. Sauer AC, Goates S, Malone A, Mogensen KM, Gewirtz G, Sulz I, et al. Prevalence of malnutrition risk and the impact of nutrition risk on hospital outcomes: results from nutritionDay in the U.S.
JPEN J Parenter Enteral Nutr 2019. doi: 10.1002/jpen.1499. [Epub ahead of print].

8. Kang MC, Kim JH, Ryu SW, Moon JY, Park JH, Park JK, et al. Prevalence of malnutrition in hospitalized patients: a multicenter cross-sectional study. J Korean Med Sci 2018;33:e10.

9. O'Shea E, Trawley S, Manning E, Barrett A, Browne V, Timmons S. Malnutrition in hospitalised older adults: A multicentre observational study of prevalence, associations and outcomes. J Nutr Health Aging 2017;21:830-6.

10. Young J, Robinson M, Chell S, Sanderson D, Chaplin S, Burns E, et al. A prospective baseline study of frail older people before the introduction of an intermediate care service. Health Soc Care Community 2005;13:307-12.

11. World Health Organization. World report on ageing and health [Internet]. Geneva: World Health Organization; 2015 [cited 2018 Dec 19]. Available from: http://apps.who.int/iris/bitstream/handle/ 10665/186463/9?sequence $=1$.

12. Compilation of the social security laws. Title XVIII: health insurance for the aged and disabled, [Internet]. Woodlawn (MD): U.S. Social Security Administration; 2018 [cited 2018 Mar 14]. Available from: https://www.ssa.gov/OP_Home/ssact/title18/1800.htm.

13. Compilation of the social security laws. Title XIX: grants to states for medical assistance programs [Internet]. Woodlawn (MD): U.S. Social Security Administration; 2018 [cited 2018 Mar 14]. Available from: https://www.ssa.gov/OP_Home/ssact/title19/1900.htm.

14. Fetter RB, Thompson JD. Inventors of DRGs look at PPS now. Interview by Marybeth Burke. Hospitals 1992;66:136-8.

15. Guterman S, Dobson A. Impact of the medicare prospective payment system for hospitals. Health Care Financ Rev 1986;7:97-114.

16. Kosecoff J, Kahn KL, Rogers WH, Reinisch EJ, Sherwood MJ, Rubenstein LV, et al. Prospective payment system and impairment at discharge. The 'quicker-and-sicker' story revisited. JAMA 1990;264:1980-3.

17. Mechanic R. Post-acute care--the next frontier for controlling Medicare spending. N Engl J Med 2014;370:692-4.

18. Young J, Gladman JR, Forsyth DR, Holditch C. The second national audit of intermediate care. Age Ageing 2015;44:182-4.

19. Yang Z, Norton EC, Stearns SC. Longevity and health care expenditures: the real reasons older people spend more. J Gerontol B Psychol Sci Soc Sci 2003;58:S2-10.

20. The Medicare Payment Advisory Commission (MedPAC). PostAcute Care [Internet]. Washington, DC: The Medicare Payment Advisory Commission (MedPAC); 2017 [cited 2018 Dec 19]. Available from: http://medpac.gov/-research-areas-/post-acute-care.

21. Vetter NJ. Intermediate care: policy before evidence. BMJ 2005;331:320.

22. Chen LK, Hwang SJ. Intermediate care: the key to health care for older people. Taiwan Geriatr Gerontol 2007;3:1-11.

23. Melis RJF, Rikkert MGMO, Parker SG, van Eijken MIJ. What is intermediate care?: an international consensus on what constitutes intermediate care is needed. BMJ 2004;329:360-1.

24. Parker G, Bhakta P, Katbamna S, Lovett C, Paisley S, Parker S, et al. Best place of care for older people after acute and during subacute illness: a systematic review. J Health Serv Res Policy 2000;5:176-89.

25. Young J. The development of intermediate care services in Eng- 
land. Arch Gerontol Geriatr 2009;49 Suppl 2:S21-5.

26. O’Reilly J, Lowson K, Young J, Forster A, Green J, Small N. A cost effectiveness analysis within a randomised controlled trial of post-acute care of older people in a community hospital. BMJ 2006;333:228.

27. Walsh B, Steiner A, Pickering RM, Ward-Basu J. Economic evaluation of nurse led intermediate care versus standard care for postacute medical patients: cost minimisation analysis of data from a randomised controlled trial. BMJ 2005;330:699.

28. National Health Service (NHS Benchmarking Network). National audit of intermediate care report 2012 [Internet]. Manchester: National Health Service (NHS Benchmarking Network); 2012 [cited 2018 Dec 19]. Available from: https://static1.squarespace. com/static/58d8d0ffe4fcb5ad94cde63e/t/58f08fc3c534a563177e6 6b6/1492160477083/naic-report-2012.pdf.

29. Ackerly DC, Grabowski DC. Post-acute care reform--beyond the ACA. N Engl J Med 2014;370:689-91.

30. Burke RE, Juarez-Colunga E, Levy C, Prochazka AV, Coleman EA, Ginde AA. Rise of post-acute care facilities as a discharge destination of US hospitalizations. JAMA Intern Med 2015;175:295-6.

31. Huang TY, Liang CK, Shen HC, Chen HI, Liao MC, Chou MY, et al. Gait speed rather than dynapenia is a simple indicator for complex care needs: a cross-sectional study using minimum data set. Sci Rep 2017;7:8418.

32. Young J, Forster A, Green J. An estimate of post-acute intermediate care need in an elderly care department for older people. Health Soc Care Community 2003;11:229-31.

33. Volpato S, Onder G, Cavalieri M, Guerra G, Sioulis F, Maraldi C, et al.; Italian Group of Pharmacoepidemiology in the Elderly Study (GIFA). Characteristics of nondisabled older patients developing new disability associated with medical illnesses and hospitalization. J Gen Intern Med 2007;22:668-74.

34. McCusker J, Kakuma R, Abrahamowicz M. Predictors of functional decline in hospitalized elderly patients: a systematic review. J Gerontol A Biol Sci Med Sci 2002;57:M569-77.

35. Covinsky KE, Palmer RM, Fortinsky RH, Counsell SR, Stewart AL, Kresevic D, et al. Loss of independence in activities of daily living in older adults hospitalized with medical illnesses: increased vulnerability with age. J Am Geriatr Soc 2003;51:451-8.

36. Pedone C, Ercolani S, Catani M, Maggio D, Ruggiero C, Quartesan $\mathrm{R}$, et al. Elderly patients with cognitive impairment have a high risk for functional decline during hospitalization: the GIFA study.
J Gerontol A Biol Sci Med Sci 2005;60:1576-80.

37. Chou MY, Liang CK, Lin YT, Chen LK, Peng LN, Liu LK, et al. Screening postacute care needs of hospital inpatients in Taiwan: A hospital-based study. J Clin Gerontol Geriatr 2012;3:132-5.

38. Green J, Young J, Forster A, Mallinder K, Bogle S, Lowson K, et al. Effects of locality based community hospital care on independence in older people needing rehabilitation: randomised controlled trial. BMJ 2005;331:317-22.

39. Garåsen H, Windspoll R, Johnsen R. Intermediate care at a community hospital as an alternative to prolonged general hospital care for elderly patients: a randomised controlled trial. BMC Public Health 2007;7:68.

40. Chen LK, Chen YM, Hwang SJ, Peng LN, Lin MH, Lee WJ, et al. Effectiveness of community hospital-based post-acute care on functional recovery and 12-month mortality in older patients: a prospective cohort study. Ann Med 2010;42:630-6.

41. Lee WJ, Cheng YY, Liu CY, Peng LN, Liu LK, Chen LK. Dosedependent effect of rehabilitation in functional recovery of older patients in the post-acute care unit. Arch Gerontol Geriatr 2012;54:e290-3.

42. Lee WJ, Peng LN, Cheng YY, Liu CY, Chen LK, Yu HC. Effectiveness of short-term interdisciplinary intervention on postacute patients in Taiwan. J Am Med Dir Assoc 2011;12:29-32.

43. Peng LN, Chen WM, Chen CF, Huang CK, Lee WJ, Chen LK. Survival benefits of post-acute care for older patients with hip fractures in Taiwan: a 5-year prospective cohort study. Geriatr Gerontol Int 2016;16:28-36.

44. Liu ME, Chou MY, Liang CK, Ho CA, Lin YT, Lo YK, et al. No adverse impact of depressive symptoms on the effectiveness of postacute care service: a multicenter male-predominant prospective cohort study. J Chin Med Assoc 2014;77:38-43.

45. Becker M, Boaz T, Andel R, DeMuth A. Predictors of avoidable hospitalizations among assisted living residents. J Am Med Dir Assoc 2012;13:355-9.

46. Lee WJ, Chou MY, Peng LN, Liang CK, Liu LK, Liu CL, et al. Predicting clinical instability of older patients in post-acute care units: a nationwide cohort study. Geriatr Gerontol Int 2014;14:267-72.

47. Peng LN, Lu WH, Liang CK, Chou MY, Chung CP, Tsai SL, et al. Functional outcomes, subsequent healthcare utilization, and mortality of stroke postacute care patients in Taiwan: a nationwide propensity score-matched study. J Am Med Dir Assoc 2017;18:990.e7-990.e12. 